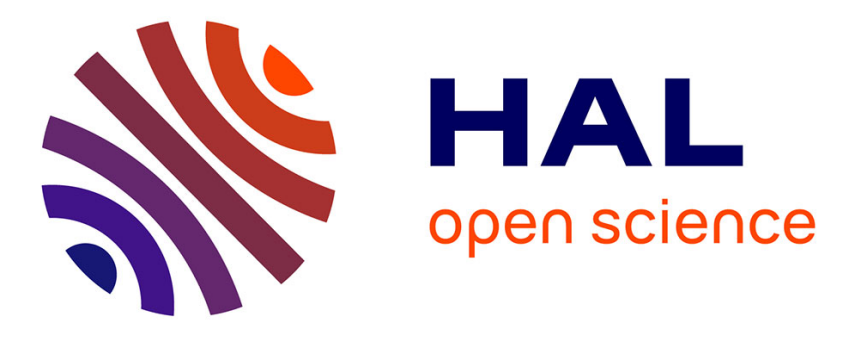

\title{
Metabolic energy-independent mechanism of internalization for the cell penetrating peptide penetratin
}

Ofelia Maniti, Élise Blanchard, Germain Trugnan, Antonin Lamazière, Jesus Ayala-Sanmartin

\section{To cite this version:}

Ofelia Maniti, Élise Blanchard, Germain Trugnan, Antonin Lamazière, Jesus Ayala-Sanmartin. Metabolic energy-independent mechanism of internalization for the cell penetrating peptide penetratin. International Journal of Biochemistry and Cell Biology, 2012, 10.1016/j.biocel.2012.02.010 . hal-02359763

\section{HAL Id: hal-02359763 \\ https://hal.science/hal-02359763}

Submitted on 12 Nov 2019

HAL is a multi-disciplinary open access archive for the deposit and dissemination of scientific research documents, whether they are published or not. The documents may come from teaching and research institutions in France or abroad, or from public or private research centers.
L'archive ouverte pluridisciplinaire HAL, est destinée au dépôt et à la diffusion de documents scientifiques de niveau recherche, publiés ou non, émanant des établissements d'enseignement et de recherche français ou étrangers, des laboratoires publics ou privés. 


\title{
Metabolic energy-independent mechanism of internalization for the cell
}

\author{
penetrating peptide penetratin
}

Ofelia Maniti, Elise Blanchard, Germain Trugnan, Antonin Lamazière and Jesus Ayala-

Sanmartin*

\begin{abstract}
Affiliations
CNRS, UMR 7203, Laboratoire des Biomolécules, Groupe N. J. Conté, Paris, France.

École Normale Supérieure, Département de Chimie, 24 rue Lhomond, 75005 Paris France.

Université Pierre et Marie Curie Paris 6, 4 Place Jussieu, 75005 Paris, France.

* Correspondence at. UMR 7203, Laboratoire des Biomolécules, Université Pierre et Marie Curie Paris 6, 4 Place Jussieu, BP 182, 75005 Paris, France. Tel: 33+1 4427 3842. Fax: 33+1 44277150. Email: jesus.ayala-sanmartin@upmc.fr.
\end{abstract}

\section{Abstract}

Cellular uptake of vector peptides used for internalization of hydrophilic molecules into cells is known to follow two different pathways: direct translocation of the plasma membrane and internalization by endocytosis followed by release into the cytosol. These pathways differ in their energy dependence. The first does not need metabolic energy while the second requires metabolic energy. Herein we used erythrocytes and plasma membrane vesicles to study membrane perturbations induced by the cell penetrating peptide penetratin. The results show that cell penetrating peptides are able to be internalized by two metabolic energy-independent pathways: direct crossing of the plasma membrane and endocytosis-like mechanisms. The last mechanism involves the induction of membrane negative curvature resulting in invaginations that mimic the endosomal uptake in the absence of ATP. This new mechanism called "physical endocytosis" or "self-induced endocytosis" might explain different data concerning the independence or dependence on metabolic energy during cellular uptake and reveals the autonomous capacity of peptides to induce their internalization. 
Keywords: Membrane invagination/ metabolic energy/ penetratin/ penetrating peptide/ physical endocytosis/ self-induced endocytosis.

\section{Introduction}

Cell penetrating peptides (CPPs) and protein transduction domains (PTDs) have been developed to introduce active molecules into cells (Mae and Langel, 2006; Murriel and Dowdy, 2006). The understanding of the uptake mechanisms of CPPs is essential to develop more efficient vectors for therapeutic purposes. Penetratin, a peptide derived from the third helix of Antennapedia transcription factor homeodomain was described, with Tat, as the first vectors to successfully carry active molecules inside cells (Joliot et al., 1991; Perez et al., 1994).

It has become clear that CPPs cellular delivery results from both: direct plasma membrane crossing, and internalization by endocytic routes with the posterior endosomal release into the cytosol (Duchardt et al., 2007; Lundin et al., 2008). Moreover, it was shown that cationic and amphipathic peptides use different endocytic routes for cellular uptake (Lundin et al., 2008) indicating a sequence-structure dependence for the mechanism of internalization. The two "mechanisms" can be separated according to their metabolic energy dependence. The energydependent mechanisms are usually associated with different types of endocytosis, and the energy-independent mechanisms with the direct translocation through the phospholipid bilayer. Recently, it was shown that at low peptide concentration $(1 \mu \mathrm{M})$ penetratin was mainly internalized by direct plasma membrane translocation whereas at higher concentration $(10 \mu \mathrm{M})$ a GAG-mediated endocytic pathway was responsible of cellular uptake (Alves et al., 2011). However, data from cellular studies are frequently ambiguous for two reasons. First, it must be considered that a peptide able to translocate directly through the plasma membrane, is able to associate to other internal membranes. Therefore, these punctuated structures observed by optical microscopy can be interpreted as endosomes. Secondly, it is difficult to completely block the metabolic energy at a physiological temperature to be sure that penetration follows only direct plasma membrane translocation.

For these reasons, several membrane models have been used for biophysical studies and membrane crossing quantification. CPPs seem to be able to translocate through the membrane of giant vesicles (GUV of several $\mu \mathrm{m}$ ) (Persson et al., 2004a; Thoren et al., 2000), but unable to cross the membrane of small vesicles $(100 \mathrm{~nm})$ unless an electrostatic (or $\mathrm{pH})$ driving membrane potential is present (Madani et al., 2011; Terrone et al., 2003). Membrane models 
also allowed the demonstration of peptide induced phospholipid phase separation and membrane deformations such as tubulation by induction of negative curvature (Alves et al., 2008; Lamaziere et al., 2007; Lamaziere et al., 2006; Lamaziere et al., 2008). However these models are quite simple compared to a biological membrane with complex lipid composition, containing different proteins, glycosylated molecules and presenting lateral and transverse asymmetries.

Elaborated cellular models for the plasma membrane are therefore required. Recently, several groups showed that giant vesicles from the plasma membrane are useful models for cellular studies. These vesicles, called giant plasma membrane vesicles (GPMVs) or plasma membrane spheres (PMS), depending on the method used for their production, are void of cellular organelles and their spherical morphology is adapted to observe peptide induced membrane deformations. Their overall composition represents that of the plasma membrane. They don't have cytoskeleton elements, are able to experiment protein and phospholipid phase separation (Baumgart et al., 2007; Keller et al., 2009; Lingwood et al., 2008) and importantly for this study, they are depleted of metabolic energy. Recently these plasma membrane derived vesicles were used to study the binding kinetics and penetration of several CPPs (Amand et al., 2011; Saalik et al., 2011).

In this study we used PMS as a model to observe the interaction of penetratin and other basic peptides with the plasma membrane. We show that penetratin is able to use two metabolic energy-independent pathways to be internalized into PMS. The first mechanism is the well known direct translocation of the plasma membrane, and the second involves the induction of endocytic-like membrane invaginations ("physical endocytosis" or "self-induced endocytosis"). The importance of these mechanisms for physiological cell penetration is discussed.

\section{Materials and methods}

\subsection{Materials.}

The peptides penetratin (Pen: RQIKIWFQNRRMKWKK), Carboxyfluorescein-labelled penetratin (CF-Pen), nona-arginine (R9), RW9 (RWRRWWRRW) and RW16 (RRWRRWWRRWWRRWRR) where synthesized and purified as previously described (Lamaziere et al., 2007; Lamaziere et al., 2008). DiI (C16) was from Invitrogene and di-4ANEPPDHQ was obtained from Dr Leslie M. Loew (Connecticut, USA).

\subsection{Erythrocytes ghosts and erythrocyte fusion.}


$8.5 \mathrm{ml}$ of human blood were mixed with the anticoagulant ACD. Erythrocytes were washed three times in buffer A (HEPES $10 \mathrm{mM} \mathrm{pH} \mathrm{7.4,} \mathrm{NaCl} 150 \mathrm{mM}$ ) by successive centrifugations at $150 \mathrm{x} \mathrm{g}$ for 10 minutes.

The preparation of erythrocytes ghosts was performed as described by Steck and Kant (Steck and Kant, 1974). Briefly, concentrated erythrocytes were lysed by addition of 40 volumes of lysis buffer (Sodium phosphate $5 \mathrm{mM}, \mathrm{pH}$ 8). The ghosts were recovered by centrifugation at $22000 \mathrm{x}$ g for 20 minutes and washed three times with lysis buffer.

Erythrocytes fusion was performed by electrofusion. $50 \mu 1$ of erythrocytes were added to an electroporation cuvette $(1 \mathrm{~mm})$ containing $100 \mu \mathrm{l}$ of PBS buffer and they were compacted by centrifugation at $100 \mathrm{x}$ g for 2 minutes. Electrofusion was performed by two $0.15 \mathrm{~ms}$ impulsions of $1100 \mathrm{~V}$ spaced by $5 \mathrm{~s}$ with a Bio-Rad electroporator. The electrofused erythrocytes were conserved at $4^{\circ} \mathrm{C}$ until use.

\subsection{Plasma membrane spheres (PMS).}

MDCK cells were cultured in DMEM media in standard conditions as previously described (Ayala-Sanmartin et al., 2004). Briefly, The cells were incubated in DMEM medium supplemented with decomplemented foetal calf serum (10\%) and antibiotics (penicillin and streptomycin) at $37^{\circ} \mathrm{C}$ in humidified atmosphere and $\mathrm{CO}_{2}(5 \%)$ in $75 \mathrm{~cm}^{2}$ flasks. The plasma membrane spheres were obtained by the method described in (Lingwood et al., 2008). Prior to PMS induction, near confluence cell cultures were washed with PBS. Then, to induce the swelling of the plasma membrane, the cultures were incubated overnight with $1 \mathrm{ml}$ of PMS buffer (1.5 mM CaCl $2,1.5 \mathrm{mM} \mathrm{MgCl}_{2}, 5 \mathrm{mM}$ HEPES, $1 \mathrm{mg} \mathrm{ml}^{-1}$ glucose in PBS pH 7.4). After PMS formation, $1 \mathrm{ml}$ of PMS buffer was added and the cells were agitated gently for one minute. Then, the $2 \mathrm{ml}$ of buffer containing the PMS was recovered and centrifuged in an eppendorf tube at $1000 \mathrm{rpm}$ for 15 minutes. The PMS were resuspended in $100 \mu 1$ of PBS and conserved at $4^{\circ} \mathrm{C}$ for several hours. The data presented in this work was performed with 8 different PMS preparations.

\subsection{PMS and erythrocytes treatments and incubations.}

All preparations for microscopic observation were performed on $\mu$-slide 8 well slides from Ibidi containing $160 \mu \mathrm{l}$ of freshly prepared erythrocytes ghosts, fused erythrocytes or PMS. For DiI labelling, DiI stock solution (500X) in ethanol was diluted in PBS. Then, $8 \mu$ l were added for a final concentration of $10 \mu \mathrm{M}$. For labelling with di-4-ANEPPDHQ, a stock solution $(200 \mathrm{mM})$ in ethanol was diluted in PBS. Then, $20 \mu \mathrm{l}$ were added for a final 
concentration of $5 \mu \mathrm{M}$. Unless specified, the peptides Pen, CF-Pen, RW9, R9 and RW16 were added in $10 \mu \mathrm{l}$ for a final concentration of $10 \mu \mathrm{M}$.

\subsection{Phase contrast microscopy.}

The phase contrast microscopy was performed with a Zeiss Axiovert 2000M inverted microscope. Images were captured with a CCD camera (Cool SNAP HQ) controlled with Metamorph software (Roper Scientific).

\section{6. Confocal microscopy.}

All the fluorescence confocal images were acquired with a TCS SP2 laser-scanning spectral system (Leica, Wetzlar, Germany) attached to a Leica DMR inverted microscope (Platform Imagerie cellulaire et tissulaire, IFR65). Optical sections were recorded with a 63/1.4 or 100/1.4 immersion objectives. Laser scanning confocal images were collected, and analyzed using the online Scan Ware software. Figures compilation was accomplished using ImageJ software (NIH). For CF-Pen and di-4-ANEPPDHQ, the samples were excited at $488 \mathrm{~nm}$ (Ar ion laser). The fluorescence emission was collected using band-pass of 500-540 nm for CFPen and 610-650 nm for di-4-ANEPPDHQ. For the experiments using CF-Pen and di-4ANEPPDHQ simultaneously the laser intensity and the PMTs were set to avoid the detection of the CF-Pen at 610-650 nm by using a standard with CF-Pen and no di-4-ANEPPDHQ. For the experiments with DiI and CF-Pen, the samples were sequentially excited. Firstly by excitation at $488 \mathrm{~nm}$ for CF-Pen, and collecting fluorescence emission using a band-pass of $500-560 \mathrm{~nm}$. A second excitation for DiI (543nm with a HeNe laser) was followed, and the emission was collected with a band-pass of 560-650 $\mathrm{nm}$. In this case the excitation of each fluorophore was independent precluding bleed-through between channels.

\section{Results}

In precedent studies we demonstrated that several CPPs are able to induce invaginations in simple and pure lipid model membranes (GUV) (Lamaziere et al., 2007; Lamaziere et al., 2009; Lamaziere et al., 2008). We called this phenomenon "physical endocytosis". Herein, we show experiments demonstrating that these peptides are also able to deform cellular plasma membranes. The cellular plasma membrane is morphologically very complex and dynamic. The cytosol is filled with intracellular membranous structures complicating the interpretation of microscopic observations. Therefore, to study CPP-membrane interactions on a spherical 
plasma membrane with no internal membranous organelles, we decided to use erythrocytes and Plasma Membrane Spheres (PMS).

\subsection{Cell penetrating peptides interaction with erythrocytes.}

Erythrocytes are membrane organelles-free cells but are small and discoid in shape.

Therefore, to facilitate microscopic observation of spherical structures, in a first series of experiments we used erythrocytes ghosts stained with the fluorescent molecule di-4ANEPPDHQ. This dye is not fluorescent in aqueous solution and has been used to characterize membrane fluidity (Jin et al., 2006). The erythrocytes ghosts were labelled homogeneously with the probe. Figure 1a and c show confocal slices of ghosts with diameters close to 4-5 $\mu \mathrm{m}$. The most evident phenomenon observed after the addition of penetratin and nona-arginine (R9) to the ghosts was their aggregation (Fig. 1b). Membrane aggregation induced by several CPPs has been previously reported in model membranes (Lamaziere et al., 2007; Persson et al., 2004b; Persson et al., 2001). The ghosts are quite small and did not attach to the surface of the chamber making their observation and monitoring difficult by confocal microscopy. Nevertheless, we were able to observe some internal membranes in the ghosts incubated with R9 (Figure 1d). In order to improve the microscopic observation, we increased the size of the erythrocytes by electrofusion. The efficiency of erythrocytes fusion was low and their shape was quite variable. They present very frequently complex forms with bud like structures which are probably rests of fused erythrocytes unable to acquire the spherical shape (Fig. 1e). Figure 1f shows a 3D reconstruction from confocal microscopy slices in which a tubular like structure was formed in fused erythrocytes incubated with the peptide penetratin. However, the low efficiency of erythrocyte fusion and their particular morphology resulted in ambiguity of interpretation of some membranous structures associated with the plasma membrane. Then, we used the Plasma Membrane Spheres (PMS) as cellular model for further analysis.

\subsection{Penetratin crosses the plasma membrane bilayer of PMS.}

Plasma membrane derived vesicles have been partially characterized by several groups. Their molecular composition is very close to that of the parent cells. They contain membrane proteins such as Thy1, Lyn and FceRI, IP17, and several transmembrane and GPI anchored proteins. The phosphatidylcholine and phosphatidylethanolamine contents are unperturbed but phosphatidylserine asymmetry is partially lost. They are able to experiment membrane 
domains separation, and they conserve their impermeability. They are depleted in both, ATP and cortical actin (Baumgart et al., 2007; Levental et al., 2011; Lingwood et al., 2008). In a first series of experiments we incubated PMS with fluorescent penetratin to a final concentration of $5 \mu \mathrm{M}$. In this conditions, and as previously described by other groups using GPMVs (Amand et al., 2011; Saalik et al., 2011), we observed that penetratin was internalized into the PMS (Figure 2). Careful analysis of confocal images showed that besides a diffuse labelling (Fig. 2a-c), spots of fluorescence associated to the membrane were observed (Figure 2d-f). The diffuse signal in the vesicular lumen demonstrates that penetratin is able to cross the plasma membrane and accumulates inside (Fig 2a-c). This accumulation was observed previously for different peptides (Amand et al., 2011; Saalik et al., 2011) and is probably due to peptide association to negatively charged macromolecules present in the lumen such as proteins and RNA. This association precludes the backward diffusion to the extracellular milieu resulting in accumulation in the cytosolic compartment. The characterization of CPP internalization on GPMVs was published elsewhere (Amand et al., 2011; Saalik et al., 2011) thus, we focused our experiments on the characterization of the membrane deformations induced by penetratin (Fig. 2d-f).

\subsection{Penetratin induces membrane invaginations in PMS.}

Considering that CPPs internalization by membrane crossing was observed at 1-5 $\mu \mathrm{M}$ concentrations for GPMVs (Amand et al., 2011; Saalik et al., 2011) and cells (Alves et al., 2011), but the glycan-mediated endoctosis was observed at $10 \mu \mathrm{M}$ penetratin (Alves et al., 2011), we decided to increase the peptide concentration to $10 \mu \mathrm{M}$ for the next experiments. PMS were observed by phase contrast microscopy in the absence or presence of penetratin. After peptide addition, we observed a time-dependent accumulation of dark structures inside the vesicles (Figure 3a). To better characterize these structures we prepared di-4ANEPPDHQ-labelled PMS. Figure $3 b$ is a stack of confocal slices of control PMS showing that PMS are free of internal membranes. The advantage of this probe is that the membrane and the peptide are excited at the same time and the emission signals are captured simultaneously. Figures $3 \mathrm{c}$ and $3 \mathrm{~d}$ show the accumulation of penetratin in structures originated in the plasma membrane that grew towards the vesicular lumen (see also supplementary Fig S1). The confocal 3D projections of PMS incubated with penetratin in supplementary videos S1 and S2 illustrate the high number and length of these penetratin induced invaginations. The co-localisation of the peptide with the membrane was revealed by the light turquoise colour (Fig. 3c and 3d). However, to avoid the risk of imaging artefacts 
when capturing images from penetratin and membranes simultaneously, in a second series of experiments we labelled the membranes with the fluorescent probe DiI before the incubation with CF-Pen (supplementary video S3). In these conditions excitation and emission of each probe were independent. As shown in figure $3 \mathrm{e}$ at short times of penetratin incubation, the membrane tubes and penetratin label correlated. At longer times the membrane tubes growth towards the centre of the vesicle allowing movement inside the PMS lumen. Due to the delay of images capture, movements inside the vesicle made difficult to observe a perfect colocalization of penetratin with membranes (Supplementary video S4). However, colocalisation was clear for many of the internal membrane-peptide structures (Figure 3f, see also supplementary figure S1). The penetratin induced membrane invaginations of the PMS membranes was observed in PMS with or without the two probes indicating that the formation of invaginations depends on the peptide and not on the probes used (Figure 4).

\subsection{Other small CPPs are able to induce membrane invaginations.}

Three other arginine rich CPPs were tested for their capacity to induce membrane invaginations on PMS; the small positively charged peptide nona-arginine (R9), and two amphipathic peptides of different length (RW9 and RW16). The long amphipathic peptide RW16 did not induce internal membrane tubulation. Figure 5 shows images of internal tubes observed after incubation with R9 and the short amphipathic peptide RW9. Interestingly, the form of these tubes was sometimes different from those observed with penetratin. The membrane structures observed after penetratin incubation were always compact and twisted. After incubation with R9 and RW9 we observed both, compacted membrane tubes and other invaginations forming well shaped large tubes (Fig. 5). As for penetratin, the invaginations were observed about 20 minutes after peptide addition.

\section{Discussion}

In this paper, we studied the internalization and the morphological membrane perturbations of basic peptides on plasma membrane spheres (PMS). Plasma membrane vesicles have been obtained by different cellular treatments. The difference between GPMV and PMS resides in the method used for their production. Giant plasma membrane vesicles (GPMV) are obtained by treatments with chemical agents such as formaldehyde and DTT, acetone or ethanol (Baumgart et al., 2007), while PMS are obtained by long incubation of cells with PBS-glucose without addition of other organic molecules. To avoid the risk of lipid solubilisation by organic solvents or protein modifications by formaldehyde and DTT, we preferred to use 
PMS obtained with a buffer free of organic chemicals. The experiments presented here demonstrate an endocytic-like mechanism of internalization for penetratin into biological vesicles. The capacity of penetratin to directly translocate into GPMVs has been recently shown by two different groups (Amand et al., 2011; Saalik et al., 2011) and was corroborated herein with PMS. In both cases, the authors showed that the GPMVs conserve their membrane integrity.

Our experiments showed direct membrane translocation at low peptide concentration (1-5 $\mu \mathrm{M})$ and tube formation at higher concentration $(10 \mu \mathrm{M})$. However, the performed confocal experiments are not adapted for the fine study of the concentration dependence of peptide activity. The vesicles must be attached to the slide and the peptide is added concentrated in a small volume to avoid movement of the vesicles. The peptide diffuses from one point on the well towards the bottom were the vesicles are located. Therefore, the local concentration close to the vesicles when membrane translocation and tubular growth starts is not precisely measured. These arguments are also valid for the time dependency study because the peptide action on the membrane depends also on the peptide diffusion time. However, the time for microscopic observation of peptides effects shown here are in the same range (about 10 minutes after peptide addition) that those previously published for the GPMVs (Amand et al., 2011; Saalik et al., 2011).

Herein we describe a novel mechanism for CPP internalization on plasma membrane vesicles involving membrane invaginations. With R9 and penetratin, membrane invaginations in erythrocyte ghosts and fused erythrocytes were observed although at a very low frequency. Penetratin, R9 and RW9 peptides were also able to induce membrane invaginations on PMS suggesting that this mechanism is shared by some basic penetrating peptides in plasma membranes of different cell types. However, the membrane invaginations were not observed with the peptide RW16 indicating that all the basic peptides did not share this property. The absence of invaginations induced by RW16 could be related to its size and capacity to form a long amphipathic helix (16 residues) that favours its insertion into the membrane and the formation of small pores (Lamaziere et al., 2007). The capacity of basic CPPs to form tubes was previously observed in membrane models (Lamaziere et al., 2007; Lamaziere et al., 2010; Menger et al., 2003) but to our knowledge this is the first time that the CPP induced tubulation is observed with biological membranes. However, this capacity to invaginate membranes has been observed for other peptides and protein domains such as the shiga toxin (Romer et al., 2007) and I bar domains (Saarikangas et al., 2009). Membrane invaginations are due to negative curvature induction. Peptides' capacity to favour membrane negative 
curvature was previously demonstrated in GUVs (Lamaziere et al., 2007; Lamaziere et al., 2009; Lamaziere et al., 2008). For penetratin and peptides such as R9 negative curvature would be produced by compaction of phospholipids after the association of arginine residues with the phospholipids head groups on the external leaflet of the vesicles as previously suggested for giant unilamellar vesicles (Lamaziere et al., 2007). A second possible mechanism to induce negative curvature would be by removing lipids from the outer layer of the membrane. However, this mechanism seems improbable and requires the formation of micelles-like structures originated from the outer leaflet of the bilayer. Once invaginations are initiated, changes in membrane tension would allow tubes to grow inside the vesicles (Lamaziere et al., 2010). The shape of invaginations seemed to be different for the different peptides. R9 and RW9 showed thicker tubes and at low frequency compared to those induced by penetratin that were more frequent, thinner and apparently twisted. This would certainly be related to the peptide sequence and therefore to the structural capacities of the different peptides. R9 and RW9 are very basic and short, RW9 is amphipathic while penetratin is longer, structurally more complex and conformationally versatile (Caesar et al., 2006; Magzoub et al., 2002; Magzoub et al., 2001; Maniti et al., 2010; Persson et al., 2004b). It is tempting to speculate that penetratin bind the membranes by different points on its surface favouring membrane bridging that results in the extension of thinner and twisted tubes.

In living cells a tubular growth in such a high number and membrane extension has never been observed. This is probably due to two important factors. First, the observed tubular growth is probably due to the absence of the cortical cytoskeleton in the PMS. This fact would allow the initial step necessary for membrane flexibility and negative curvature induction. The cortical cytoskeleton which is dynamically stable in erythrocytes might block membrane invaginations explaining the very low frequency of tubes observed in fused erythrocytes and ghosts. The second factor is that in a living cell, after membrane invagination, the endocytic vesicles are rapidly cut from the plasma membrane by dynamin. This step is not present in the PMS and therefore, the tubular extension of the membrane invaginations is allowed. These explanations are supported by the work with shiga toxin showing the stabilization of membrane invaginations in mutants with no dynamin or after blocking actin function (Romer et al., 2007).

At $10 \mu \mathrm{M}$ peptide concentration, we rarely observed diffuse fluorescent penetratin in the cytosol at the same time that membrane invaginations. In cells it has been shown that CPPs are internalized by direct membrane translocation at low concentration $(1 \mu \mathrm{M})$ and that at 
higher concentration they are preferentially internalized by GAG-mediated endocytosis (Alves et al., 2011). In our conditions $(10 \mu \mathrm{M})$, penetratin favoured physical endocytosis indicating that at high concentrations peptide-peptide interactions at the membrane surface are necessary to form the tubes. Our observations also indicate that the peptide did not cross the membrane of tubes. Two related factors might be involved in the apparent absence of membrane translocation from tubes; curvature and $\mathrm{pH}$ gradient. It has been shown that membrane translocation depends on the size (curvature) of the vesicles. In almost flat membranes such as GUVs of several $\mu \mathrm{m}$ in diameter $(10-30 \mu \mathrm{m})$, penetratin is able to cross the membrane (Persson et al., 2004a; Thoren et al., 2000). In highly curved membranes (100 $\mathrm{nm}$ LUVs) the efficient penetration requires ionic potential or $\mathrm{pH}$ acidification (Madani et al., 2011; Terrone et al., 2003). Therefore, the absence of acidification in highly curved structures would decrease the probability of peptide membrane translocation. In metabolically normal cells, CPP escape from the endosomes is thought to be driven by the acidification of the endosome. Therefore when "physical endocytosis" occurs in normal cells, the posterior traffic of the vesicles and their fusion with endosomes would result in acidification of the lumen and peptide release into the cytosol.

Does Physical endocytosis occur in living cells? We tentatively suggest that the response to this question is yes. The intrinsic structural capacity of the peptide to induce negative curvature might be independent of the metabolic endocytosis. The independence of the peptide uptake and several endocytic pathways is illustrated by the fact that TAT uptake does not depend on clathrin, caveolae or dynamin (Wadia et al., 2004). However, passive internalisation of membrane associated peptide during different endocytic processes is not excluded. If these hypotheses are true, we can predict that besides the normal dynamic movements of the plasma membrane, the peptides would be able to provoke inward vesiculation resulting not only in peptide internalization but also in an increase of the rate of endocytosis of other molecules. This prediction is supported by the fact that, the CPPs TAT and penetratin increase the neutral dextran fluid phase cellular uptake (Amand et al., 2008; Wadia et al., 2004).

\section{Conclusion}

In conclusion, our data show that "physical" or "self-induced" endocytosis is a novel mechanism for penetratin cellular internalization. This mechanism is defined as the capacity of a peptide to produce endosome like invaginations without participation of metabolic energy. As shown in the schematic representation of figure 6, the main differences between 
the model of physical endocytosis and the biological endocytic pathways is the absence of a metabolic energy-dependent driving force for the induction of inward vesiculation. The driving force for physical endocytosis results from the peptide self-capacity to induce negative curvature.

\section{Acknowledgments}

We thank Philippe Fontanges and Romain Morichon of the platform Imagerie cellulaire et tissulaire (IFR65 Hôpital Tenon, France) for the confocal microscopy facility, and Sandrine Sagan for critical reading of the manuscript and useful discussions.

\section{References}

Alves ID, Bechara C, Walrant A, Zaltsman Y, Jiao CY, Sagan S. Relationships between membrane binding, affinity and cell internalization efficacy of a cell-penetrating peptide: penetratin as a case study. PLoS One 2011; 6: e24096.

Alves ID, Goasdoue N, Correia I, Aubry S, Galanth C, Sagan S, et al. Membrane interaction and perturbation mechanisms induced by two cationic cell penetrating peptides with distinct charge distribution. Biochim Biophys Acta 2008; 1780: 948-59.

Amand HL, Bostrom CL, Lincoln P, Norden B, Esbjorner EK. Binding of cell-penetrating penetratin peptides to plasma membrane vesicles correlates directly with cellular uptake. Biochim Biophys Acta 2011; 1808: 1860-7.

Amand HL, Fant K, Norden B, Esbjorner EK. Stimulated endocytosis in penetratin uptake: effect of arginine and lysine. Biochem Biophys Res Commun 2008; 371: 621-5.

Ayala-Sanmartin J, Cavusoglu N, Masliah J, Trugnan G. Homodimerization of Annexin 2. Role of the $\mathrm{N}$-terminal tail and modulation of membrane aggregation properties. Annexins 2004; 1: 19-25. Baumgart T, Hammond AT, Sengupta P, Hess ST, Holowka DA, Baird BA, et al. Large-scale fluid/fluid phase separation of proteins and lipids in giant plasma membrane vesicles. Proc Natl Acad Sci U S A 2007; 104: 3165-70.

Caesar CE, Esbjorner EK, Lincoln P, Norden B. Membrane interactions of cell-penetrating peptides probed by tryptophan fluorescence and dichroism techniques: correlations of structure to cellular uptake. Biochemistry 2006; 45: 7682-92.

Duchardt F, Fotin-Mleczek M, Schwarz H, Fischer R, Brock R. A comprehensive model for the cellular uptake of cationic cell-penetrating peptides. Traffic 2007; 8: 848-66.

Jin L, Millard AC, Wuskell JP, Dong X, Wu D, Clark HA, Loew LM. Characterization and application of a new optical probe for membrane lipid domains. Biophys J 2006; 90: 2563-75. Joliot A, Pernelle C, Deagostini-Bazin H, Prochiantz A. Antennapedia homeobox peptide regulates neural morphogenesis. Proc Natl Acad Sci U S A 1991; 88: 1864-8.

Keller H, Lorizate M, Schwille P. PI(4,5)P2 degradation promotes the formation of cytoskeleton-free model membrane systems. Chemphyschem 2009; 10: 2805-12.

Lamaziere A, Burlina F, Wolf C, Chassaing G, Trugnan G, Ayala-Sanmartin J. Non-metabolic membrane tubulation and permeability induced by bioactive peptides. PLoS ONE 2007; 2: e201. Lamaziere A, Chassaing G, Trugnan G, Ayala-Sanmartin J. [Transduction peptides: structuralfunctional analyses in model membranes]. J Soc Biol 2006; 200: 229-33.

Lamaziere A, Chassaing G, Trugnan G, Ayala-Sanmartin J. Tubular structures in heterogeneous membranes induced by the cell penetrating peptide penetratin. Commun Integr Biol 2009; 2: 223-4. Lamaziere A, Maniti O, Wolf C, Lambert O, Chassaing G, Trugnan G, et al. Lipid domain separation, bilayer thickening and pearling induced by the cell penetrating peptide penetratin. Biochim Biophys Acta 2010; 1798: 2223-30.

Lamaziere A, Wolf C, Lambert O, Chassaing G, Trugnan G, Ayala-Sanmartin J. The homeodomain derived peptide Penetratin induces curvature of fluid membrane domains. PLoS ONE 2008; 3: e1938. 
Levental I, Grzybek M, Simons K. Raft domains of variable properties and compositions in plasma membrane vesicles. Proc Natl Acad Sci U S A 2011; 108: 11411-6.

Lingwood D, Ries J, Schwille P, Simons K. Plasma membranes are poised for activation of raft phase coalescence at physiological temperature. Proc Natl Acad Sci U S A 2008; 105: 10005-10.

Lundin P, Johansson H, Guterstam P, Holm T, Hansen M, Langel U, S ELA. Distinct uptake routes of cell-penetrating peptide conjugates. Bioconjugate chemistry 2008; 19: 2535-42.

Madani F, Peralvarez-Marin A, Graslund A. Liposome Model Systems to Study the Endosomal Escape of Cell-Penetrating Peptides: Transport across Phospholipid Membranes Induced by a Proton Gradient. Journal of drug delivery 2011; 2011: 897592.

Mae M, Langel U. Cell-penetrating peptides as vectors for peptide, protein and oligonucleotide delivery. Curr Opin Pharmacol 2006; 6: 509-14.

Magzoub M, Eriksson LE, Graslund A. Conformational states of the cell-penetrating peptide penetratin when interacting with phospholipid vesicles: effects of surface charge and peptide concentration. Biochim Biophys Acta 2002; 1563: 53-63.

Magzoub M, Kilk K, Eriksson LE, Langel U, Graslund A. Interaction and structure induction of cellpenetrating peptides in the presence of phospholipid vesicles. Biochim Biophys Acta 2001; 1512: 7789.

Maniti O, Alves I, Trugnan G, Ayala-Sanmartin J. Distinct behaviour of the homeodomain derived cell penetrating peptide penetratin in interaction with different phospholipids. PLoS One 2010; 5: e15819.

Menger FM, Seredyuk VA, Kitaeva MV, Yaroslavov AA, Melik-Nubarov NS. Migration of poly-Llysine through a lipid bilayer. J Am Chem Soc 2003; 125: 2846-7.

Murriel CL, Dowdy SF. Influence of protein transduction domains on intracellular delivery of macromolecules. Expert Opin Drug Deliv 2006; 3: 739-46.

Perez F, Lledo PM, Karagogeos D, Vincent JD, Prochiantz A, Ayala J. Rab3A and Rab3B carboxyterminal peptides are both potent and specific inhibitors of prolactin release by rat cultured anterior pituitary cells. Mol Endocrinol 1994; 8: 1278-87.

Persson D, Thoren PE, Esbjorner EK, Goksor M, Lincoln P, Norden B. Vesicle size-dependent translocation of penetratin analogs across lipid membranes. Biochim Biophys Acta 2004a; 1665: 14255 .

Persson D, Thoren PE, Lincoln P, Norden B. Vesicle membrane interactions of penetratin analogues. Biochemistry 2004b; 43: 11045-55.

Persson D, Thoren PE, Norden B. Penetratin-induced aggregation and subsequent dissociation of negatively charged phospholipid vesicles. FEBS Lett 2001; 505: 307-12.

Romer W, Berland L, Chambon V, Gaus K, Windschiegl B, Tenza D, et al. Shiga toxin induces tubular membrane invaginations for its uptake into cells. Nature 2007; 450: 670-5.

Saalik P, Niinep A, Pae J, Hansen M, Lubenets D, Langel U, et al. Penetration without cells:

Membrane translocation of cell-penetrating peptides in the model giant plasma membrane vesicles. $\mathbf{J}$

Control Release 2011; 153: 117-25.

Saarikangas J, Zhao H, Pykalainen A, Laurinmaki P, Mattila PK, Kinnunen PK, et al. Molecular mechanisms of membrane deformation by I-BAR domain proteins. Curr Biol 2009; 19: 95-107.

Steck TL, Kant JA. Preparation of impermeable ghosts and inside-out vesicles from human erythrocyte membranes. Methods in enzymology 1974; 31: 172-80.

Terrone D, Sang SL, Roudaia L, Silvius JR. Penetratin and related cell-penetrating cationic peptides can translocate across lipid bilayers in the presence of a transbilayer potential. Biochemistry 2003; 42 : 13787-99.

Thoren PE, Persson D, Karlsson M, Norden B. The antennapedia peptide penetratin translocates across lipid bilayers - the first direct observation. FEBS Lett 2000; 482: 265-8.

Wadia JS, Stan RV, Dowdy SF. Transducible TAT-HA fusogenic peptide enhances escape of TATfusion proteins after lipid raft macropinocytosis. Nature medicine 2004; 10: 310-5. 
Figure legends

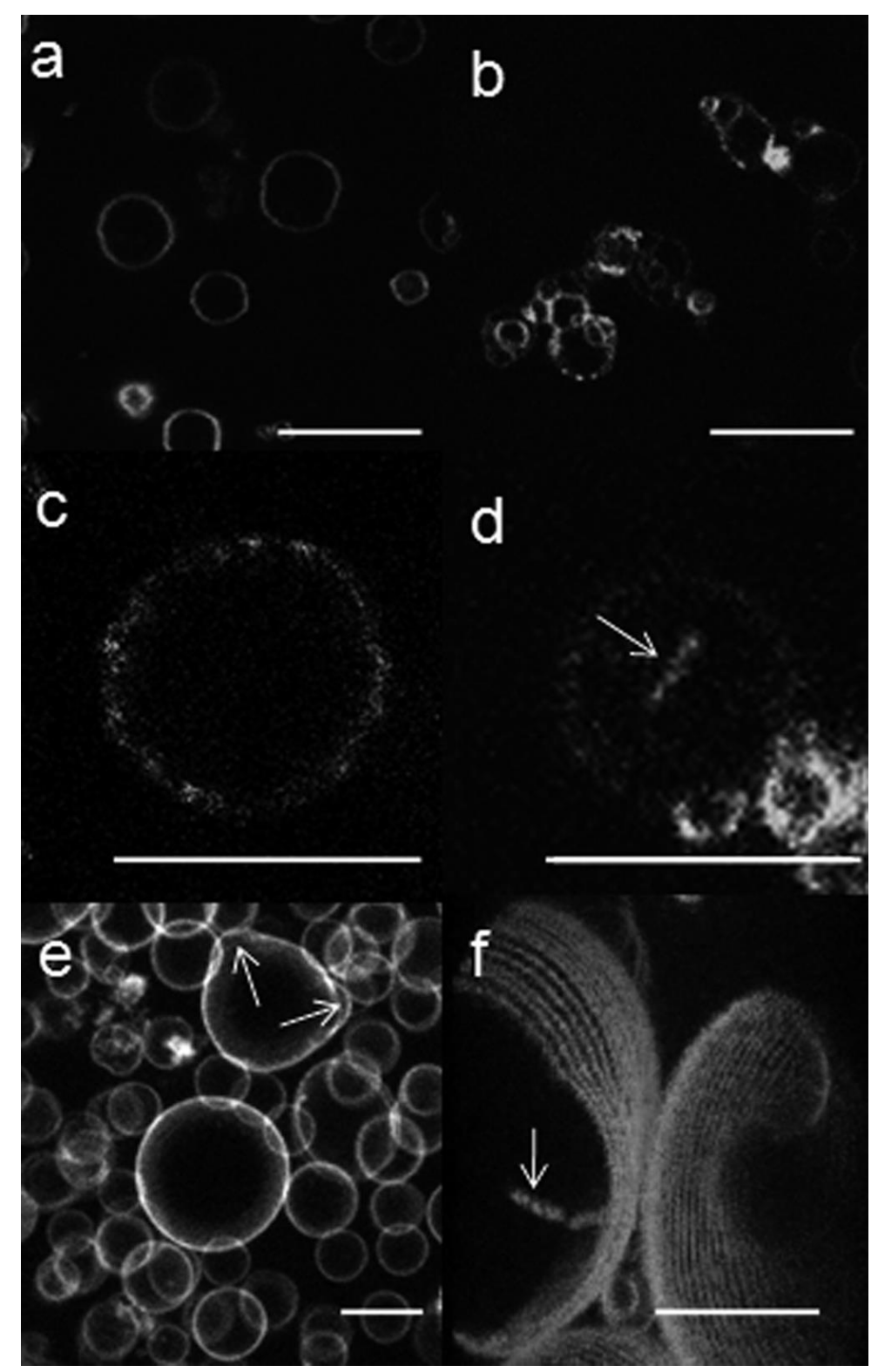

Figure 1. Plasma membrane perturbations in erythrocytes incubated with cell penetrating peptides. Erythrocytes were stained with the membrane probe di-4-ANEPPDHQ, and incubated with R9 or penetratin. Confocal slices of erythrocytes ghosts before (a) and 15 minutes after R9 addition (b). Notice the peptide mediated ghosts' aggregation. Confocal slices of an erythrocyte ghost (c) and a ghost 20 minutes after R9 addition (d). Notice the tubular structure (arrow) inside the erythrocyte ghost. Projections of confocal slices stack of two fused erythrocytes (e). Notice the bud-like structures (arrows) in the upper fused erythrocyte. Projections of confocal slices stack of two fused erythrocytes incubated with penetratin (f). A tube inside the fused erythrocyte at the left is shown (arrow). Scale bars a, b, e and f; $10 \mu \mathrm{m}, \mathrm{c}$ and d; $5 \mu \mathrm{m}$. 

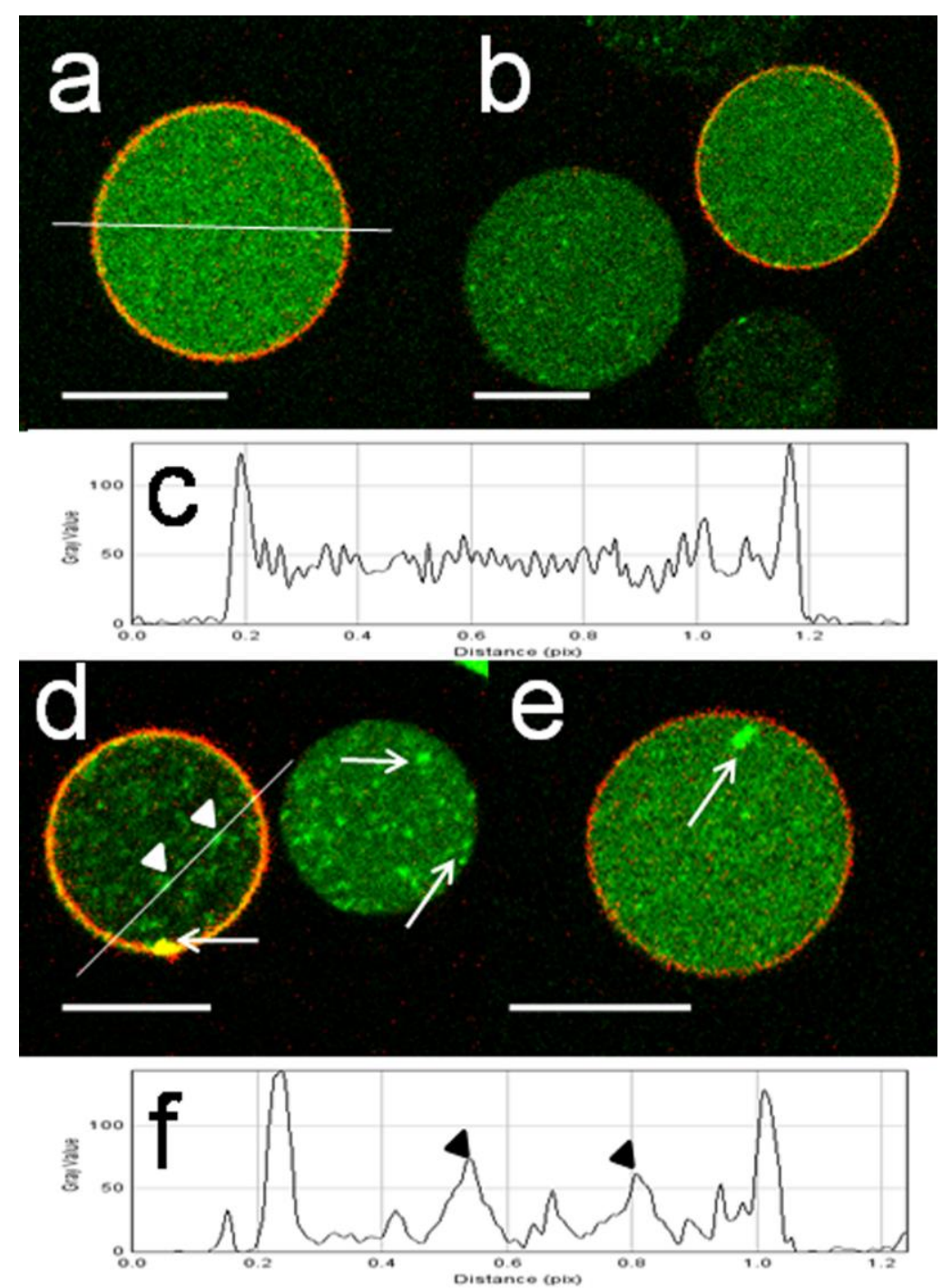

Figure 2. Direct translocation of penetratin through the plasma membrane of PMS. Confocal slices of PMS showing penetratin internalization into PMS incubated with $5 \mu \mathrm{M}$ of CF-Pen for 20 (a), and 25 minutes (b). (c) Shows the density profile of the line crossing the PMS in (a). Notice the accumulation of homogeneous fluorescence in the lumen of the vesicle. PMS incubated with CF-Pen for 40 (d) and 35 minutes (e). (f) Shows the density profile of the line crossing the PMS in (d). Notice the inhomogeneous fluorescence in the lumen of the vesicle.PMS labelled with DiI in red and CF-Pen in green. Images are representative of two independent experiments. Arrows indicate spots of fluorescent penetratin associated with membranes, arrowheads the corresponding fluorescence of the section in (d) and the profile (f). Scale bars $10 \mu \mathrm{m}$. 


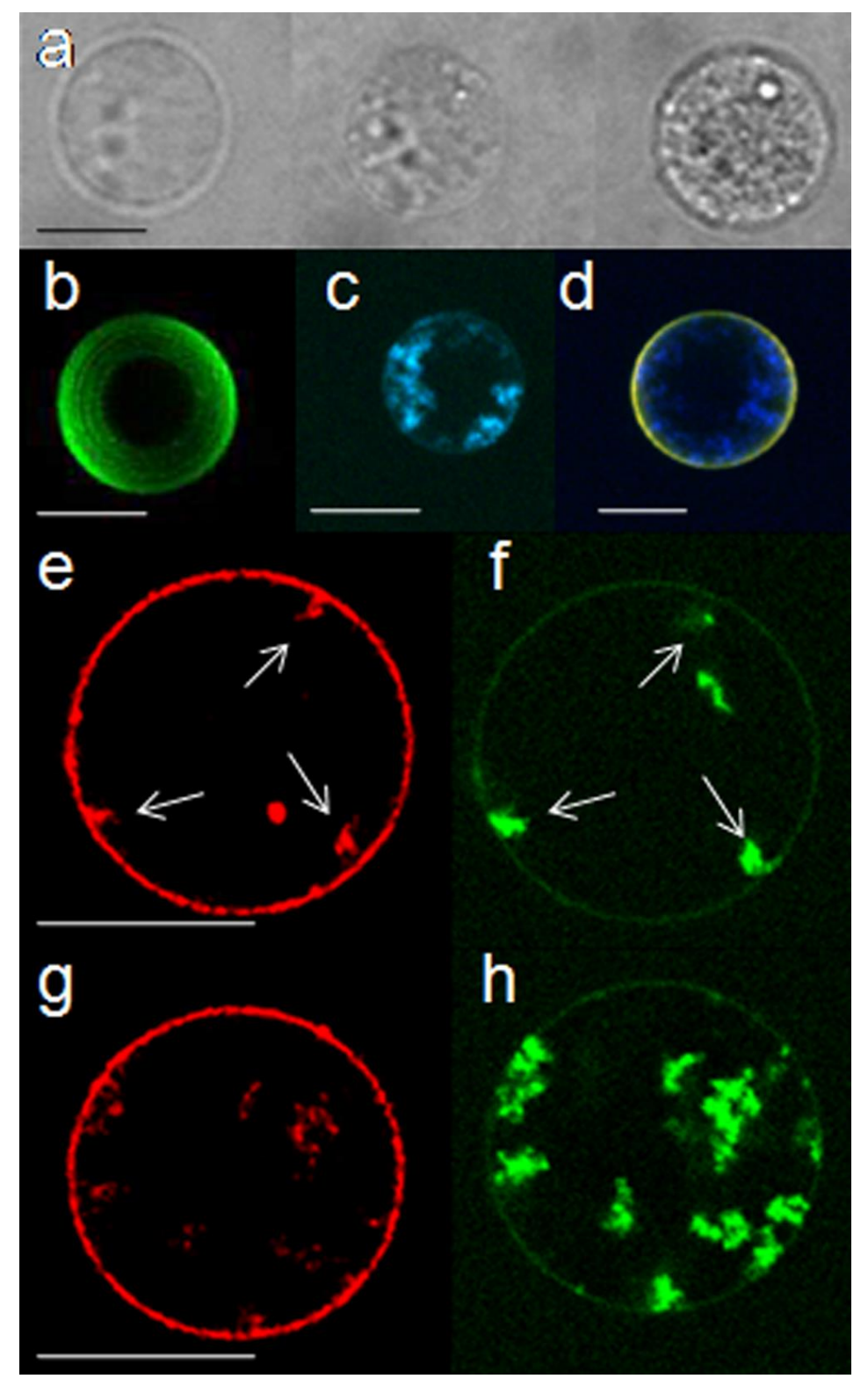

Figure 3. Penetratin induces membrane invaginations in PMS. Phase contrast images of a PMS incubated with penetratin observed at 10, 45, and 60 minutes after peptide addition (a). Projection of confocal slices of a PMS labelled with di-4-ANEPPDHQ before penetratin addition (b). Notice that the PMS does not contain membranes in its lumen. Projections of confocal slices of two different PMS labelled with di-4-ANEPPDHQ at 50 minutes (c) and 60 minutes (d) after penetratin addition. Membrane is green in (c) and yellow in (d) and CF-Pen is blue. Turquoise colour indicates colocalisation of membrane and peptide (b). Confocal slices of one PMS at 25 minutes (e,f) and 35 minutes (g,h) after CF-Pen addition. Membrane (red) was labelled with DiI (e,g) and CF-Pen is green $(f, h)$. Arrows indicate the co-localisation of membrane and penetratin. Images are representative of four (a-d) and two (e-h) independent experiments. Scale bars $10 \mu \mathrm{m}$. See also supplementary videos S1-4 and supplementary figure S1. 


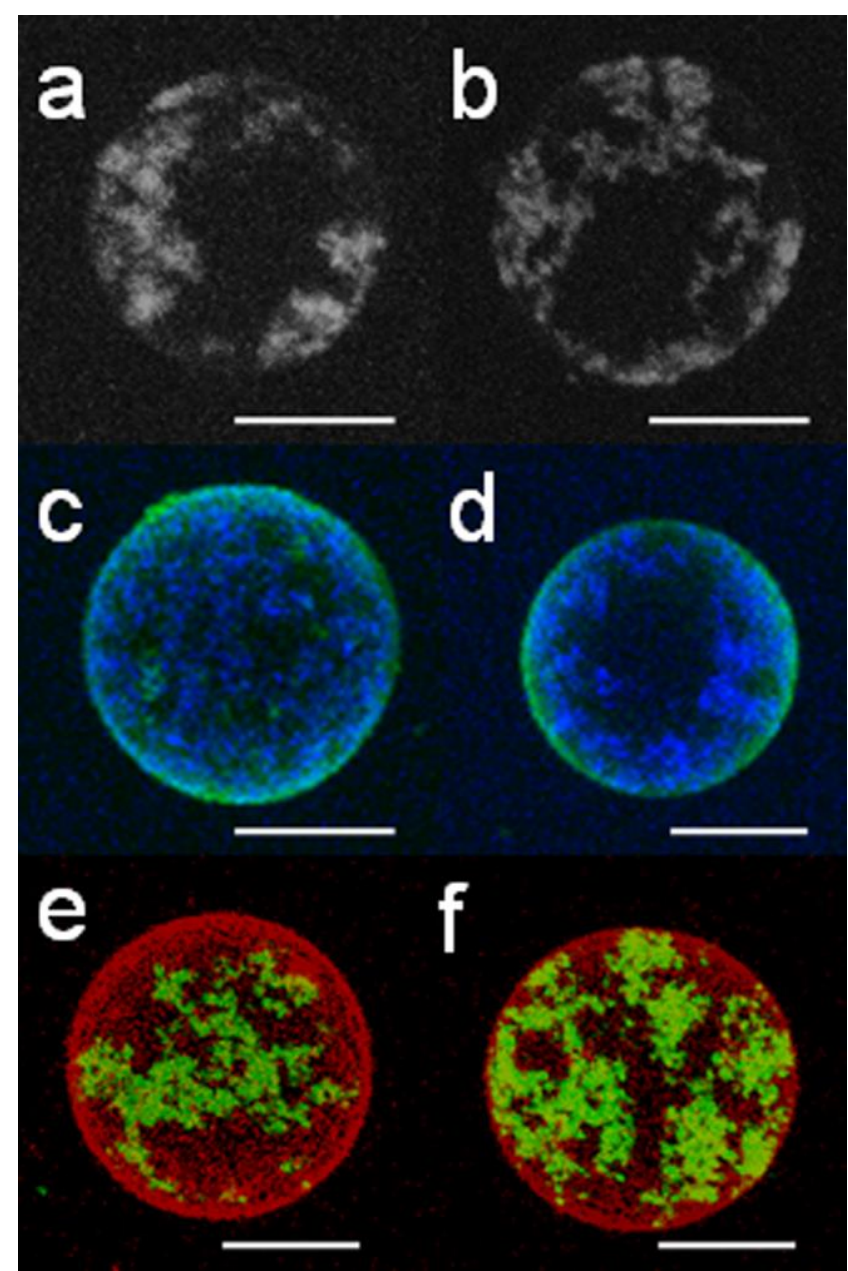

Figure 4. Penetratin induced membrane invaginations in PMS are independent of membrane labelling. Projections of confocal slices of PMS showing the whole penetratin-induced internal tubular network. Unlabelled PMS (a,b) incubated with CF-Pen. PMS labelled with di-4-ANEPPDHQ in green and CFPen in blue (c,d). PMS labelled with DiI in red and CF-Pen in green (e,f). Images are representative of two (a,b,e,f) and four (c,d) independent experiments. All images were captured between 70 to 90 minutes after penetratin incubation. Scale bars $10 \mu \mathrm{m}$. See also supplementary videos S1-4.

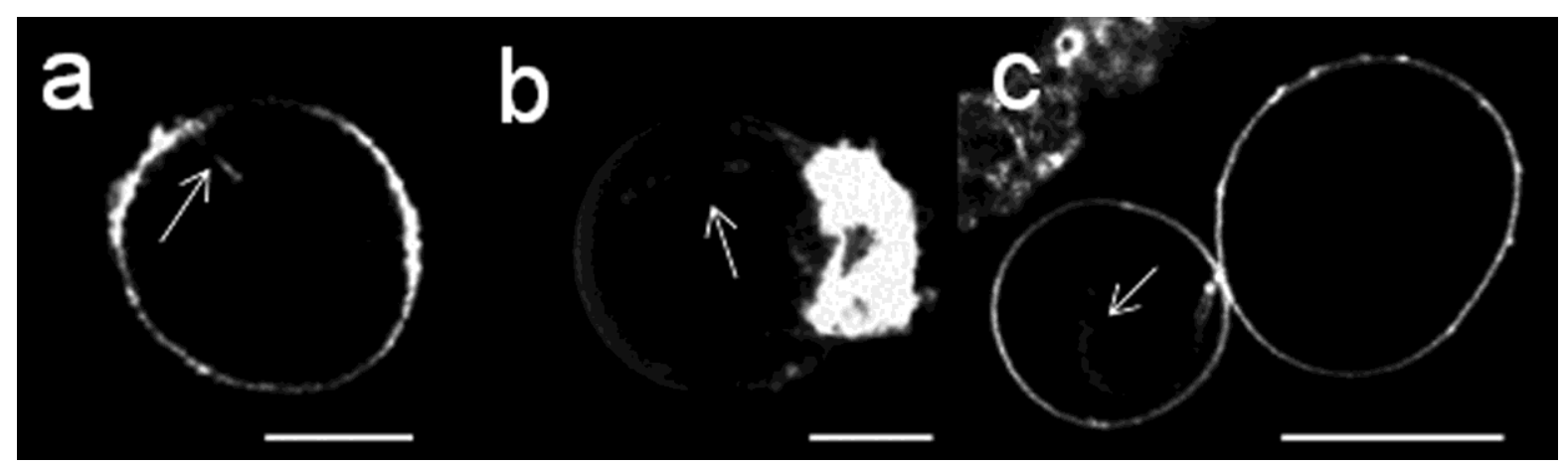

Figure 5. Small arginine rich peptides form tubular invaginations. PMS labelled with di-4-

ANEPPDHQ were incubated with R9 and RW9 peptides. (a) Confocal slice of a PMS after 25 minutes of incubation with R9 showing an internal tube (arrow) (see also supplementary video S6). (b) Confocal projection of a PMS after 120 minutes of incubation with R9 showing an internal tube (arrow) (see also supplementary video S5). The fluorescent saturated mass on the right side of the PMS is a rest of the cell that produced the vesicle. (c) Confocal slice of a PMS after 130 minutes of incubation with RW9 showing a tube inside the PMS at the left (arrow). Scale bars $10 \mu \mathrm{m}$. 


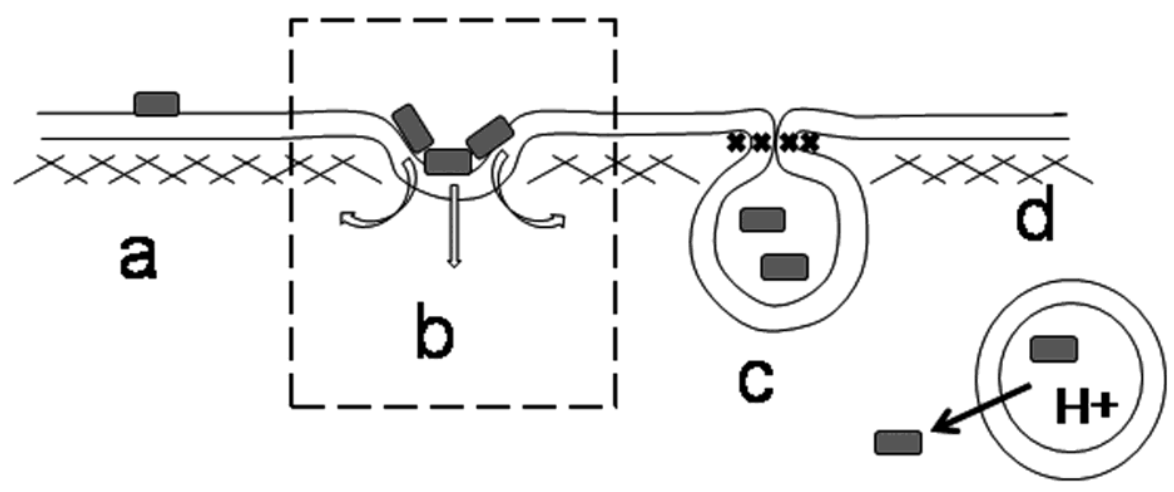

Figure 6. Model for energy-independent CPP cellular uptake and differences with metabolic endocytosis. The first step necessary for physical endocytosis is peptide binding to phospholipids (a). Notice that glycan binding of a peptide will result in normal "receptor"-mediated endocytosis. If the peptide lacks information to induce negative curvature, phospholipid binding will result also in normal endocytosis. The crucial step for physical endocytosis (b, in the box) is the induction of membrane curvature driven by the peptide itself without cellular metabolic energy (peptide action is symbolized by arrows) local absence (or particular dynamics) of cytoskeleton (crossed lines) will allow the first step for membrane invagination. In normal endocytosis this step is mediated by coating proteins such as clathrin and by membrane movements due to the cytoskeleton. The separation of the vesicle from the membrane is performed by proteins such as dynamin ( $\mathbf{x}$, in c). In PMS the absence of this step allows membrane tubulation instead of vesiculation. After fusion of endosomal vesicles with endosomes the peptide will translocate into the cytosol thanks to endosome acidification (d). 